\title{
FACTORES PSICOPATOLÓGICOS DE RIESGO EN INTENTOS DE SUICIDIO
}

\section{PSYCHOPATHOLOGICAL RISK FACTORS IN SUICIDE ATTEMPTS}

\author{
Lorena Cecilia López Steinmetz \\ Consejo Nacional de Investigaciones Cientificas y Técnicas (CONICET). Argentina \\ Departamento de Docencia e Investigación, Hospital Psiquiátrico Dr. Néstor M. Sequeiros, Jujuy. \\ Argentina
}

\begin{abstract}
Resumen: Este estudio caracterizó el factor psicopatológico de riesgo en intentos suicidas asistidos en el servicio de urgencia de un hospital general de Jujuy, Argentina. El estudio fue de tipo epidemiológico, de diseño descriptivo transversal. Las fuentes de datos fueron los registros de asistencias psicológicas durante 2007-2008 y 2011-2012. Del total de intentos suicidas $(N=481)$, el 19.54\% había intentado suicidarse anteriormente, mientras que el $28.07 \%$ tenía diagnóstico de trastorno mental, de los cuales el 56.30\% recibía tratamiento al momento del intento suicida. Trastornos del ánimo, especialmente depresivos, y Trastornos relacionados con sustancias fueron los diagnósticos más frecuentes. Se hallaron diferencias significativas según marcadores demográficos (sexo-edad) que deben ser consideradas en investigaciones futuras. Los resultados mostraron que el factor psicopatológico de riesgo suicida es mucho menos prevalente en la casuística local comparado con reportes en poblaciones foráneas. Los hallazgos relativizan la importancia del factor psicopatológico de riesgo en intentos suicidas.
\end{abstract}

Palabras clave: intento de suicidio; factores de riesgo; psicopatología; autolisis; urgencias médicas

\begin{abstract}
This study characterized the psychopathological risk factor in suicide attempts assisted in the emergency service of a general hospital in Jujuy, Argentina. The study was of the epidemiological type, of transversal-descriptive design. The data sources were psychological assists records during 2007-2008 and 2011-2012. Of total suicide attempts $(N=481), 19.54 \%$ had attempted suicide previously, while $28.07 \%$ had diagnosis of mental disorder, of which $56.30 \%$ were receiving treatment at the time of suicide attempt. Mood disorders, especially depressives, and Substance-related disorders were the most frequent diagnoses. Significant differences according to demographic markers (sex-age) that should be considered in future studies were found. The results showed that the psychopathological suicide risk factor is much less prevalent in the local casuistry compared to reports in foreign populations. The findings question the importance of psychopathological risk factor in suicide attempts.
\end{abstract}

Keywords: suicide, attempted; risk factors; psychopathology; autolysis; emergencies

\section{Introducción}

La Organización Mundial de la Salud (OMS) reconoce al suicidio como un importante problema social y de salud pública con tendencia creciente, que en 2012 produjo 804.000 muertes en todo el mundo (OMS, 2014). Se estima que por cada muerte por suicidio otras 20 personas realizan uno o más intentos suicidas (Diekstra \& Gulbinat, 1993; OMS, 2014), los que pueden tener consecuencias físicas y psíquicas de gravedad (OMS, 2014). Para la prevención del suicidio es fundamental conocer y comprender los factores asociados con un mayor riesgo de suicidio e intentos suicidas
(Teti, Rebok, Rojas, Grendas, \& Daray, 2014).

Bajo la denominación factor psicopatológico de riesgo suicida se suelen describir aspectos de la salud mental, características psiquiátricas, psicológicas y del comportamiento personal, frecuentemente asociados con un incremento del riesgo de realizar intentos suicidas o de consumar suicidio. Los intentos suicidas previos en la historia del paciente son señalados como el factor de riesgo que predice con más claridad la posibilidad de un futuro suicidio (Beautrais, 2001; Haukka, Suominen, Partonen, \& Lönngvist, 2008; Hawton \& Van Heeringen, 2009; Suominen, Isometsä, Suokas, Haukka, Achte, \& Lönngvist, 2004; Teti et al., 2014; Yoshimasu, Kiyo-

Correspondencia: Lorena Cecilia López Steinmetz. Hospital Psiquiátrico Dr. Néstor M. Sequeiros, Jujuy. Argentina.

Correo Electrónico:steinramirez@hotmail.com 
hara, Miyashita, \& The Stress Research Group of the Japanese Society for Hygiene, 2008) o de intentos de suicidio (Hishinuma et al., 2017; Ribeiro, Franklin, Fox, \& Bentley, 2016). La presencia de trastornos mentales también es indicada como uno de los mayores predictores de intentos de suicidio (Nock, Hwang, Sampson, \& Kessler, 2010), cuyo riesgo aumenta cuanto mayor es el número de diagnósticos psiquiátricos comórbidos (Beautrais, Joyce, Mulder, Fergusson, Deavoll, \& Nightingale, 1996; Bolton, Pagura, Enns, Grant, \& Sareen, 2010; Kulkarni, Rao, \& Begum, 2013; Wunderlich, Bronisch, \& Wittchen, 1998). Los trastornos mentales más frecuentemente asociados con intentos suicidas son Trastornos del estado de ánimo (Beautrais, 2000; Bridge, Goldstein, \& Brent, 2006), especialmente depresivos (Teti et al., 2014) aunque también bipolares (Baldessarini, Pompili, \& Tondo, 2006), Trastornos relacionados con sustancias (Beautrais, 2000; Bridge et al., 2006; Teti et al., 2014), Trastornos de la personalidad, de ansiedad (Groholt \& Ekeberg, 2009) y Esquizofrenia (Haukka et al., 2008; Hawton, Sutton, Haw, Sinclair, \& Deeks, 2005), entre otros. Incluso se afirmó que prácticamente todos los trastornos psiquiátricos tienen un riesgo suicida aumentado, exceptuando el Retraso mental y la Demencia (Harris \& Barraclough, 1997). En cambio, hay estudios que si bien señalaron que los trastornos mentales se encuentran entre los más fuertes predictores de suicidio (Nock et al., 2009) y de conductas suicidas (Nock et al., 2010), a su vez reconocieron que debido a los altos niveles de comorbilidad psiquiátrica, poco se sabe acerca de cuáles trastornos son singularmente predictivos de la conducta suicida. Estos estudios sugirieron que la mayor parte de las asociaciones comúnmente reseñadas se deben a trastornos que predicen la aparición de pensamientos suicidas (tal sería el caso de la depresión) más que predecir la progresión de pensamientos suicidas a intentos suicidas. En tanto que en los pocos casos en que los trastornos mentales predicen el paso desde pensamientos suicidas a intentos suicidas, serían significativos los trastornos caracterizados por ansiedad/agitación (p.e.: Trastorno por estrés postraumático) y pobre control de los impulsos (p.e.: Trastorno de conducta, Trastorno por consumo de sustancias) (Nock et al., 2009, 2010). Por otra parte, en pacientes con diagnóstico de trastorno mental, la falta de tratamiento de salud mental y el incumplimiento terapéutico se rela- cionan con mayor probabilidad de realizar intento suicida (Brent et al., 1988; González Pinto et al., 2006; Ruengorn, Sanichwankul, Niwatananun, Mahatnirunkul, Pumpaisalchai, \& Patumanond, 2012) y de repetir dicha tentativa (Groholt \& Ekeberg, 2009). Al parecer, el incumplimiento terapéutico es más frecuente en determinados diagnósticos psiquiátricos, principalmente en Trastorno depresivo (Bener, Dafeeah, \& Salem, 2013; DiMatteo, Lepper, \& Croghan, 2000), Trastorno bipolar (Gaudiano, Weinstock, \& Mi1ler, 2008) y Esquizofrenia (Bener et al., 2013).

El presente trabajo forma parte una investigación más amplia sobre coyunturas desencadenantes de urgencias suicidas (López Steinmetz, n.d.). En el presente estudio se seleccionan algunas dimensiones de lo que, en su generalidad, se denomina factor psicopatológico de riesgo suicida en los antecedentes citados, para ser analizadas en casuística asistida por intento suicida. Las dimensiones del factor psicopatológico que se analizan son: antecedente de intento suicida, diagnóstico actual de trastorno mental, Tipos y Categorías diagnósticas, y acceso a tratamiento a causa del diagnóstico de trastorno mental al momento del intento suicida. La bibliografía reconoce clásicamente las distinciones entre factores y marcadores de riesgo - dependiendo de si la relación de ellos con un cambio en el riesgo de un proceso, resultado o condición sanitaria relevante es causal o no causal respectivamente $-\mathrm{y}$ entre factores de riesgo modificables y no modificables (Porta, 2014). De acuerdo a ello y dada la distribución poblacional desigual de las tasas de suicidio, los estudios epidemiológicos reconocen determinados marcadores socio-demográficos importantes del riesgo suicida. Tal es el caso del sexo y la estructura etaria que desde hace décadas son indicados como los marcadores cruciales que determinan las dimensiones del suicidio (Nock, Borges, Bromet, Cha, Kessler, \& Lee, 2008; Polewka et al., 2004), y por ese motivo también son analizados en la presente investigación. En relación a ello, nuestros resultados previos indican que al parecer la casuística asistida por intento suicida en la provincia de Jujuy posee características diferentes respecto de lo descripto en casuísticas foráneas en cuanto a marcadores de riesgo suicida, tanto demográficos (López Steinmetz, 2017) como temporales (López Steinmetz, 2015). Profundizar el conocimiento de los factores y marcadores de riesgo que caracterizan a la 
población local es necesario, porque esto ayudará a identificar a los sujetos con mayor riesgo de presentar conductas suicidas, a fin de posibilitar el diseño de estrategias de prevención específicas. Visto todo lo anterior, en este trabajo se estudió casuística asistida psicológicamente por intento suicida en el servicio de urgencia de un hospital general de Jujuy, con el objetivo de caracterizarla según las dimensiones de análisis establecidas para el factor psicopatológico de riesgo y examinar dichas dimensiones en relación a los marcadores demográficos de riesgo suicida sexo y edad.

\section{Materiales y métodos}

\section{Ámbito, tipo de estudio y fuente de datos}

El Servicio de Guardia (o Servicio de Urgencia) del Hospital Pablo Soria (HPS) se escogió como ámbito de estudio debido a que es el principal hospital de la provincia de Jujuy (extremo noroeste de Argentina), y debido a que hasta julio de 2012 era el único hospital público en toda la provincia cuyo Servicio de Guardia contaba con asistencia de urgencias en salud mental. El HPS es una institución polivalente, de tercer nivel de complejidad, que asiste a pacientes de ambos sexos desde 15 años de edad.

El estudio fue epidemiológico, de diseño descriptivo transversal, con tratamiento cuantitativo de datos. Las fuentes de datos consistieron en los registros escritos de asistencias psicológicas brindadas en el Servicio de Guardia del HPS durante el periodo de estudio, delimitado de manera intencional a los bienios 2007-2008 y 2011-2012.

El protocolo de esta investigación fue evaluado y aprobado por el Comité Provincial de Bioética de Jujuy.

\section{Población, dimensiones y marcadores de riesgo analizados}

Se incluyeron los registros de todos los casos asistidos psicológicamente en el Servicio de Guardia del HPS por intento de suicidio como motivo de consulta durante el periodo de estudio. La conceptualización de intento de suicidio adoptada en esta investigación fue la que lo define como la conducta auto-infligida y potencialmente lesiva que no produce la muerte pero para la que hay una intención evidente, sea explícita o implícita, de morir (O’Carroll, Berman, Ma- ris, Moscicki, Tanney, \& Silverman, 1996). Las dimensiones del factor psicopatológico de riesgo analizadas en los registros del total de casos asistidos por intento de suicidio $(N=481)$ fueron:

- Presencia-ausencia de antecedente de intento suicida: la presencia de este antecedente comprendió a los casos que realizaron intento/s suicida/s con anterioridad a la tentativa por la cual estaban siendo asistidos.

- Presencia-ausencia de diagnóstico actual de trastorno mental: la presencia de esta dimensión contabilizó los casos en los que algún diagnóstico de trastorno mental fue explicitado en los registros de asistencias psicológicas por parte de los profesionales tratantes en el Servicio de Guardia, ya sea porque ellos efectuaron el diagnóstico durante la asistencia, o bien porque el paciente refirió (o sus familiares lo hicieron por él) haber sido diagnosticado con trastorno mental por algún profesional especializado en salud mental previo al intento suicida.

Las dimensiones del factor psicopatológico de riesgo analizadas en los registros del subtotal de casos asistidos por intento de suicidio que tenían diagnóstico de trastorno mental $(n=135)$ fueron:

- Tipos y Categorías diagnósticas: se agruparon los diagnósticos de trastorno mental en función de Tipos y Categorías diagnósticas según la clasificación y codificación del DSM- $I V$ TR (Asociación Americana de Psiquiatría, 2002). Para los casos que refirieron haber sido diagnosticados con trastorno mental previo al intento suicida pero que no conocían el Tipo diagnóstico recibido, se estableció la Categoría general "Diagnóstico desconocido" y se contabilizó un diagnóstico por cada uno de esos casos. Entre los demás casos, cuyos Tipos diagnósticos fueron conocidos, hubo algunos con diagnósticos comórbidos, por lo que la cantidad de Tipos diagnósticos registrados fue mayor que la cantidad de casos con diagnóstico de trastorno mental.

- Acceso a tratamiento a causa del diagnóstico de trastorno mental: se analizó si los pacientes que tenían diagnóstico de trastorno mental asistían a tratamiento psicológico y/o psiquiátrico (en cualquier modalidad) al momento del intento suicida. En función de ello, se contabilizó cada caso en una de tres categorías de tratamiento: $R e$ cibe tratamiento (pacientes que al momento del intento suicida asistían a tratamiento); Abandonó tratamiento (pacientes que habían iniciado tratamiento pero que previo al intento suicida lo ha- 
bían abandonado); No inició tratamiento (pacientes que nunca iniciaron tratamiento pese a haber sido diagnosticados con trastorno mental previo al intento suicida).

Los marcadores demográficos de riesgo suicida incluidos en los análisis de todas las dimensiones del factor psicopatológico de riesgo fueron:

- Sexo: analizado como variable dicotómica (femenino-masculino) ya que se estableció con referencia a parámetros o diferencias biológicas.

- Edad: analizado en franjas etarias, conformadas por estratos de 10 años de edad cada uno. El límite aparente inferior del estrato menor fue 15 años de edad debido a que en el HPS se asisten a pacientes de ambos sexos de $\geq 15$ años de edad.

\section{Análisis estadísticos}

En los análisis descriptivos se informaron modo, razón, y proporción (expresada en porcentajes). La Prueba de Chi-cuadrado $\left(\chi^{2}\right)$ se aplicó para determinar la existencia o no de asociación entre cada dimensión del factor psicopatológico y los marcadores demográficos de riesgo suicida establecidos. El nivel de significación estadística se fijó en $p<.05$. Dado que en la aplicación de la Prueba de Chi-cuadrado no debe haber más de un $20 \%$ de las celdas cuyas frecuencias teóricas $\left(f_{t}\right)$ tengan valores menores que 5 , cuando este fue el caso, el tipo de corrección aplicada dependió del tamaño de las tablas de contingencia (Álvarez Cáceres, 2007). Para los datos analizados en tablas de $2 \times 2$ no fue necesario aplicar correcciones ya que en ellas no se hallaron $f_{t}<5$. En tablas de mayor tamaño $(K x R)$ el procedimiento de corrección fue el de agrupar categorías de análisis y/o intervalos adyacentes - siempre que estas uniones tuvieran sentido en la realidad - y volver a calcular las $f_{t}$ con las nuevas tablas (Álvarez Cáceres, 2007). Pese a ello, en algunos análisis persistieron las $\mathrm{f}_{t}$ con valores menores que 5 en más del $20 \%$ de las celdas. En estos casos, se informó el resultado obtenido de $\chi^{2}$ acompañado de la leyenda " $f<5$ : $+20 \%$ " para advertir la persistencia de esta situación que debe ser tenida en cuenta al interpretar los resultados, y en caso de haberse hallado diferencias estadísticamente significativas se señalaron las principales asociaciones que influyeron en el valor observado de $\chi^{2}$. Respecto de las tablas que se presentan, cabe señalar dos aspectos. Por una parte, al informar los resultados de $\chi^{2}$ en análisis que se aplicaron las correcciones mencionadas, los grados de libertad (df) indicados corresponden a las tablas agrupadas, pero las tablas que se presentan corresponden a las que contienen las distribuciones originales y no las agrupadas. Por otra parte, para no duplicar información y también por cuestiones de espacio, no se presentan todas las tablas de contingencia utilizadas para los cálculos (por ejemplo, no se presentan tablas de tamaño $2 \times 2$ ). No obstante, todos los datos en base a los cuales se realizaron los cálculos están incluidos en las tablas que se presentan, por lo que las tabulaciones originales son deducibles de estas.

Finalmente, en los análisis de $\chi^{2}$ que se halló asociación estadísticamente significativa, se determinó la fuerza de la misma mediante el cálculo del coeficiente V de Cramer $(V)$.

\section{Resultados}

\section{Antecedente de intento suicida}

Del total de pacientes asistidos por intento de suicidio, sólo el 19.54\% había realizado uno o más intentos suicidas anteriores. La razón entre casos sin antecedente - con antecedente de intento suicida fue de 3.37 a 1 en el sexo femenino y de 5.96 a 1 en el masculino. La razón entre sexos (femenino-masculino) fue de 2.48 a 1 en casos con antecedente de intento suicida y de 1.40 a 1 en casos sin ese antecedente (ver tabla 1). Las diferencias según sexos fueron estadísticamente significativas $\left(\chi^{2}(1)=5.27, p<.05\right)$. Antecedente de intento suicida caracterizó diferencialmente al sexo femenino respecto del masculino, aunque la fuerza de la asociación hallada fue muy débil $(V=0.10)$.

El modo por edad fue 19.5 años en casos femeninos con y sin antecedente de intento suicida $\mathrm{y}$ en masculinos sin ese antecedente. En cambio, en masculinos con antecedente de intento suicida el modo por edad fue 29.5 años. Se registraron casos con antecedente de intento suicida hasta 55-64 años en el sexo femenino y hasta 3544 años en el masculino. En la composición por edad, el grupo con antecedente de intento suicida se diferenció significativamente del grupo sin ese antecedente en el conjunto ambos sexos $\left(\chi^{2}(4)=10.84, p<.05\right)$ y en el sexo masculino $\left(\chi^{2}(3)=10.92, p<.05\right)$, pero no en el femenino $\left(\chi^{2}(3)=7.30, p>.05\right)$. La fuerza de las asociacio- 
nes halladas fue de $\mathrm{V}=0.15 \mathrm{y}$ de $\mathrm{V}=0.24$ respectivamente. Entre las asociaciones principales en ambos casos, antecedente de intento suicida caracterizó diferencialmente a la franja etaria 25-34 años, y ausencia de ese antecedente a 15-24 años.

Tabla 1.

Total de casos de intento de suicidio $(N=481)$ según presencia-ausencia de antecedente de intento suicida, franja etaria y sexo

\begin{tabular}{lcccccc}
\hline \multirow{2}{*}{$\begin{array}{l}\text { Franja } \\
\text { etaria }\end{array}$} & \multicolumn{5}{c}{ Antecedente de intento suicida } \\
\cline { 2 - 7 } & Semenino & No & Sí & No & Sí & No \\
\hline $15-24$ & 28 & 133 & 10 & 86 & 38 & 219 \\
$25-34$ & 19 & 54 & 14 & 38 & 33 & 92 \\
$35-44$ & 14 & 26 & 3 & 19 & 17 & 45 \\
$45-54$ & 5 & 8 & 0 & 12 & 5 & 20 \\
$55-64$ & 1 & 3 & 0 & 4 & 1 & 7 \\
$65-74$ & 0 & 2 & 0 & 2 & 0 & 4 \\
$\mathrm{n}$ & 67 & 226 & 27 & 161 & 94 & 387 \\
\hline
\end{tabular}

Sí: presencia de antecedente de intento suicida; No: ausencia de antecedente de intento suicida.

\section{Diagnóstico actual de trastorno mental}

Del total de pacientes asistidos por intento suicida, sólo el $28.07 \%$ tenía diagnóstico de trastorno mental. La razón entre casos sin diagnóstico - con diagnóstico de trastorno mental fue de 3.07 a 1 en el sexo femenino y de 1.98 a 1 en el masculino. La razón entre sexos (femenino-masculino) fue de 1.14 a 1 en casos con diagnóstico de trastorno mental y de 1.77 a 1 en casos sin esos diagnósti$\cos$ (ver tabla 2). Las diferencias según sexos fueron estadísticamente significativas $\left(\chi^{2}(1)=4.52\right.$, $p<.05)$, aunque la fuerza de la asociación hallada fue muy débil $(V=0.10)$. La presencia de diagnóstico de trastorno mental caracterizó diferencialmente al sexo masculino respecto del femenino.

En ambos grupos de pacientes - con y sin diagnóstico de trastorno mental - y en cada sexo, el modo por edad fue 19.5 años. En la composición por edad, el grupo con diagnóstico de trastorno mental se diferenció significativamente del grupo sin esos diagnósticos en el conjunto ambos $\operatorname{sexos}\left(\chi^{2}(4)=23.38, p<.05\right)$ y en el sexo femenino $\left(\chi^{2}(3)=17.30, p<.05\right)$, pero no en el masculino $\left(\chi^{2}(3)=6.37, p>.05\right)$. La fuerza de las asociaciones halladas fue de $V=0.22$ y de $\mathrm{V}=0.24$ respectivamente. Entre las asociaciones principales en ambos casos, la presencia de diagnóstico de trastorno mental caracterizó diferen- cialmente a las franjas etarias 35-44 y 55-64 años, y la ausencia de esos diagnósticos a 15-24 años.

Tabla 2.

Total de casos de intento de suicidio $(N=481)$ según presencia-ausencia de diagnóstico actual de trastorno mental, franja etaria y sexo

\begin{tabular}{lcccccc}
\hline \multirow{2}{*}{$\begin{array}{l}\text { Franja } \\
\text { etaria }\end{array}$} & \multicolumn{5}{c}{ Demeniagnóstico de trastorno mental } \\
\cline { 2 - 7 } & Sí & No & Sí & No & Sí & No \\
\hline $15-24$ & 26 & 135 & 26 & 70 & 52 & 205 \\
$25-34$ & 21 & 52 & 19 & 33 & 40 & 85 \\
$35-44$ & 16 & 24 & 12 & 10 & 28 & 34 \\
$45-54$ & 5 & 8 & 3 & 9 & 8 & 17 \\
$55-64$ & 3 & 1 & 3 & 1 & 6 & 2 \\
$65-74$ & 1 & 1 & 0 & 2 & 1 & 3 \\
$\mathrm{n}$ & 72 & 221 & 63 & 125 & 135 & 346
\end{tabular}

Sí: presencia de diagnóstico actual de trastorno mental; No: ausencia de diagnóstico actual de trastorno mental.

\section{Tipos y Categorías diagnósticas}

Del subtotal de pacientes ambos sexos asistidos por intento suicida que tenían diagnóstico de trastorno mental $(n=135), 38$ ( 25 femeninos, 13 masculinos) refirieron haber sido diagnosticados con trastorno mental pero desconocían el Tipo diagnóstico recibido. De los 97 pacientes restantes cuyos Tipos diagnósticos fueron conocidos (47 femeninos, 50 masculinos), sólo 5 (2 femeninos, 3 masculinos) tenían trastornos mentales comórbidos (de 2 diagnósticos en cada caso). De acuerdo a ello, la cantidad de Tipos diagnósticos de trastorno mental registrados fue de 140 en el conjunto ambos sexos, de los cuales 74 se registraron en el sexo femenino y 66 en el masculino. El Tipo diagnóstico modal en el sexo femenino fue Trastorno depresivo no especificado, seguido en frecuencia por Dependencia de varias sustancias (ver tabla 3), mientras que en el masculino fue modal Dependencia del alcohol, seguido de Trastorno depresivo no especificado (ver tabla 4). En términos de Categorías diagnósticas, Trastornos del estado de ánimo y Trastornos relacionados con sustancias fueron de las más frecuentes aunque con diferentes ordenamientos en cada sexo. La Categoría establecida como Diagnóstico desconocido también estuvo entre las más frecuentes en cada sexo. A pesar de las similitudes señaladas, las distribuciones de las Categorías diagnósticas se diferenciaron significativamente 
Tabla 3.

Cantidad de diagnósticos de trastorno mental en casos de intento de suicidio sexo femenino según

Categoría-Tipo diagnóstico y franja etaria

\begin{tabular}{ccccccccc}
\hline \multirow{2}{*}{ Categoría y Tipo diagnóstico* $^{*}$} & \multicolumn{7}{c}{ Franja etaria } & \multirow{2}{c}{$\mathrm{n}$} \\
\cline { 2 - 7 } & $15-24$ & $25-34$ & $35-44$ & $45-54$ & $55-64$ & $65-74$ & \\
\hline Trast. de la conducta alimentaria & 5 & 0 & 0 & 0 & 0 & 0 & 5 \\
F50.9 & 2 & 0 & 0 & 0 & 0 & 0 & 2 \\
F50.2 & 3 & 0 & 0 & 0 & 0 & 0 & 3
\end{tabular}

Trast. de la personalidad

F60.31

F60.9

$\begin{array}{lllllll}3 & 2 & 0 & 0 & 0 & 0 & 5 \\ 3 & 1 & 0 & 0 & 0 & 0 & 4 \\ 0 & 1 & 0 & 0 & 0 & 0 & 1\end{array}$

Trast. del control de los impulsos no clasifi-

cados en otros apartados F63.9

$\begin{array}{llllllc}0 & 1 & 0 & 0 & 0 & 0 & 1 \\ 0 & 1 & 0 & 0 & 0 & 0 & 1 \\ 1 & 2 & 6 & 2 & 1 & 1 & 13 \\ 0 & 2 & 5 & 2 & 1 & 0 & 10 \\ 1 & 0 & 1 & 0 & 0 & 1 & 3\end{array}$

Trast. de ansiedad

F41.9

F41.0

Esquizofrenia y otros trast. psicóticos F29

Trast. relacionados con sustancias

F19.2x

F13.2x

Trast.deinicioenlainfancia, niñezoadolescencia F79.9

1

$\begin{array}{llllll}1 & 2 & 0 & 0 & 0 & 4 \\ 1 & 1 & 0 & 0 & 0 & 2\end{array}$

$\begin{array}{lllllll}1 & 0 & 1 & 0 & 0 & 0 & 2\end{array}$

Delirium, demencia, trast. amnésicos y

otros trast. cognoscitivos

F03

Diagnóstico desconocido

Total diagnósticos sexo femenino

$\begin{array}{lllllll}2 & 5 & 1 & 0 & 0 & 0 & 8 \\ 2 & 5 & 1 & 0 & 0 & 0 & 8\end{array}$

$\begin{array}{ccccccc}5 & 2 & 2 & 1 & 0 & 0 & 10 \\ 5 & 2 & 2 & 0 & 0 & 0 & 9 \\ 0 & 0 & 0 & 1 & 0 & 0 & 1\end{array}$

*Clasificación y codificación según DSM-IV-TR (Asociación Americana de Psiquiatría, 2002).

Tabla 4.

Cantidad de diagnósticos de trastorno mental en casos de intento de suicidio sexo masculino según Categoría-Tipo diagnóstico y franja etaria

\begin{tabular}{|c|c|c|c|c|c|c|}
\hline \multirow{2}{*}{ Categoría y Tipo diagnóstico* } & \multicolumn{5}{|c|}{ Franja etaria } & \multirow{2}{*}{$\mathrm{n}$} \\
\hline & $15-24$ & $25-34$ & $35-44$ & $45-54$ & $55-64$ & \\
\hline \multirow{2}{*}{$\begin{array}{l}\text { Trast. del control de los impulsos no } \\
\text { clasificados en otros apartados } \\
\text { F63.0 }\end{array}$} & 1 & 0 & 0 & 0 & 0 & 1 \\
\hline & 1 & 0 & 0 & 0 & 0 & 1 \\
\hline Trast. del estado de ánimo & 4 & 7 & 1 & 1 & 1 & 14 \\
\hline F32.9 & 3 & 6 & 1 & 1 & 1 & 12 \\
\hline F31.9 & 1 & 1 & 0 & 0 & 0 & 2 \\
\hline Trast. de ansiedad & 1 & 0 & 1 & 0 & 0 & 2 \\
\hline F41.9 & 0 & 0 & 1 & 0 & 0 & 1 \\
\hline F43.1 & 1 & 0 & 0 & 0 & 0 & 1 \\
\hline Esquizofrenia y otros trast. psicóticos & 3 & 2 & 5 & 2 & 1 & 13 \\
\hline F29 & 1 & 0 & 2 & 2 & 1 & 6 \\
\hline F22.00 & 0 & 0 & 1 & 0 & 0 & 1 \\
\hline F20.xx & 2 & 2 & 2 & 0 & 0 & 6 \\
\hline Trast. relacionados con sustancias & 12 & 5 & 5 & 0 & 1 & 23 \\
\hline F19.2x & 7 & 3 & 0 & 0 & 0 & 10 \\
\hline F10.2x & 5 & 2 & 5 & 0 & 1 & 13 \\
\hline Diagnóstico desconocido & 7 & 5 & 1 & 0 & 0 & 13 \\
\hline Total diagnósticos sexo masculino & 28 & 19 & 13 & 3 & 3 & 66 \\
\hline
\end{tabular}


según el sexo de los pacientes $\left(\chi^{2}(9)=23.43\right.$, $\left.p<.05\left[\mathrm{f}_{\mathrm{t}}<5:+20 \%\right]\right)$.

La fuerza de la asociación fue de $V=0.41$. Entre las asociaciones más destacadas, Trastornos de la conducta alimentaria y Trastornos de la personalidad caracterizaron diferencialmente al sexo femenino, mientras que Trastornos relacionados con sustancias, y Esquizofrenia y otros trastornos psicóticos caracterizaron diferencialmente al sexo masculino.

Las distribuciones de Tipos y Categorías diagnósticas por franjas etarias en casos femeninos se muestran en la tabla 3 y las correspondientes a casos masculinos en la tabla 4. Las distribuciones de las Categorías diagnósticas no se diferenciaron significativamente según edad de los pacientes en el conjunto ambos sexos $\left(\chi^{2}(14)=23.14, p>.05\right.$ $\left.\left[\mathrm{f}_{\mathrm{t}}<5:+20 \%\right]\right)$, en el sexo femenino $\left(\chi^{2}(14)=\right.$ $\left.23.45, p>.05\left[\mathrm{f}_{\mathrm{t}}<5:+20 \%\right]\right)$, ni en el masculino $\left(\chi^{2}(10)=9.68, p>.05\left[f_{t}<5:+20 \%\right]\right)$ analizados separadamente. Cabe mencionar que en estos cálculos de $\chi^{2}$ se excluyeron las frecuencias de las Categorías diagnósticas que en sí mismas implicaban criterios diagnósticos atinentes a la edad (Trastornos de inicio en la infancia, niñez o adolescencia, y Delirium, demencias, trastornos amnésicos y otros trastornos cognoscitivos).

\section{Acceso a tratamiento a causa del diagnóstico de trastorno mental}

Del subtotal de pacientes ambos sexos que tenían diagnóstico de trastorno mental $(n=135)$, el $56.30 \%$ recibía tratamiento a causa del diag- nóstico al momento del intento suicida, mientras que el restante $43.70 \%$ no lo recibía, ya sea porque había abandonado el tratamiento $(14.07 \%)$ o bien porque nunca lo había iniciado (29.63\%). La razón entre sexos (femenino-masculino) fue de 1.45 a 1 entre quienes recibían tratamiento, de 2.80 a 1 entre quienes lo abandonaron, y de 0.48 a 1 entre quienes no lo iniciaron (ver tabla 5). Las diferencias según sexos fueron estadísticamente significativas $\left(\chi^{2}(2)=11.19, p<.05\right)$. La fuerza de la asociación fue de $V=0.29$. Los casos femeninos se caracterizaron por recibir tratamiento pero también por haberlo abandonado, mientras que los masculinos se caracterizaron por no haber iniciado tratamiento.

Los casos que recibían tratamiento al momento del intento suicida fueron predominantes en las tres franjas etarias menores y en la mayor, tanto del grupo ambos sexos como del sexo femenino. En el sexo masculino, los casos que recibían tratamiento sólo predominaron en 25-34 y 35-44 años (tabla 5). En la composición por edad, se hallaron diferencias estadísticamente significativas en el acceso a tratamiento a causa del diagnóstico de trastorno mental en el grupo ambos $\operatorname{sexos}\left(\chi^{2}(4)=12.44, p<.05\right)$ y en el sexo femenino $\left(\chi^{2}(4)=13.05, p<.05\right)$, pero no en el masculino $\left(\chi^{2}(4)=3.95, p>.05\left[f_{t}<5:+20 \%\right]\right)$. La fuerza de las asociaciones halladas fue de $V$ $=0.21$ y de $V=0.30$ respectivamente. Entre las asociaciones destacadas en ambos casos, 15-24 años se caracterizó por recibir tratamiento, 25-34 y 35-44 años por haberlo abandonado, y 45-54 y 55-64 años por no haberlo iniciado.

Tabla 5.

Casos de intento de suicidio con diagnóstico actual de trastorno mental $(n=135)$, según acceso a tratamiento a causa del diagnóstico, sexo y franja etaria

\begin{tabular}{cccccccccc}
\hline & \multicolumn{8}{c}{ Tratamiento por el diagnóstico } \\
\cline { 2 - 9 } Franja etaria & \multicolumn{3}{c}{ Femenino } & \multicolumn{7}{c}{ Masculino } & \multicolumn{3}{c}{ Ambos sexos } \\
\cline { 2 - 10 } & Sí & Abandonó & No & Sí & Abandonó & No & Sí & Abandonó & No \\
\hline $15-24$ & 21 & 1 & 4 & 10 & 3 & 13 & 31 & 4 & 17 \\
$25-34$ & 11 & 6 & 4 & 11 & 2 & 6 & 22 & 8 & 10 \\
$35-44$ & 9 & 6 & 1 & 9 & 0 & 3 & 18 & 6 & 4 \\
$45-54$ & 2 & 1 & 2 & 0 & 0 & 3 & 2 & 1 & 5 \\
$55-64$ & 1 & 0 & 2 & 1 & 0 & 2 & 2 & 0 & 4 \\
$65-74$ & 1 & 0 & 0 & 0 & 0 & 0 & 1 & 0 & 0 \\
$\mathrm{n}$ & 45 & 14 & 13 & 31 & 5 & 27 & 76 & 19 & 40 \\
\hline
\end{tabular}

Sí: recibe tratamiento; Abandonó: abandonó tratamiento; No: no inició tratamiento. 


\section{Discusión y conclusiones}

En este estudio se encontró que aquellos pacientes que habían realizado un intento suicida con anterioridad representaban un $19.54 \%$, lo que estuvo muy por debajo de los reportes de entre el $30 \%$ (Haukka et al., 2008) y el $50 \%$ (Schmidtke et al., 1996). Los resultados sugirieron que los casos femeninos se caracterizan por tener antecedente de intento suicida. Sin embargo, al incluir el análisis del marcador demográfico edad, sugirieron que la presencia de ese tipo de antecedente caracteriza diferencialmente a la franja etaria 25-34 años del grupo ambos sexos y del sexo masculino pero no del femenino, mientras que la ausencia de ese antecedente caracteriza a 15-24 años del sexo masculino y también, aunque con fuerza menor, del grupo ambos sexos. Coincidentemente con lo hallado según sexo de los pacientes, hay estudios que informaron mayor incidencia de intentos suicidas repetidos en mujeres antes que en hombres tanto de poblaciones foráneas (Haukka et al., 2008; Scoliers, Portzky, Van Heeringen, \& Audenaert, 2009) como de poblaciones latinas (Teti et al., 2014). De acuerdo a lo hallado según edad, hay estudios que también reportaron que grupos de menos de 20 años se diferenciaron significativamente de los de mayor edad que se caracterizaron por tener tasas de repetición de intentos suicidas más altas (Scoliers et al., 2009). Tal es el caso, por ejemplo, de la franja etaria 30-40 años que fue asociada con mayor riesgo de intentos suicidas repetidos (Haukka et al., 2008). La repetición de conductas suicidas también fue asociada con tener cualquier diagnóstico de trastorno mental y, especialmente, Trastorno relacionado con el alcohol (Haukka et al., 2008), altos niveles de ansiedad, depresión severa, y más síntomas psiquiátricos (Scoliers et al., 2009). Se conformarían dos tipos de grupos, los que realizan un solo intento suicida que difieren clínicamente de aquellos con múltiples tentativas, entre los que se reportan mayores niveles de patología y de riesgo para futuros intentos de suicidio y mayor probabilidad de autolisis (Colborn et al., 2017; Forman, Berk, Henriques, Brown, \& Beck, 2004; Johnson Fridell, Ojehagen, \& Träskman-Bendz, 1996; Joiner \& Rudd, 2000; Joiner, Rudd, Rouleau, \& Wagner, 2000; Miranda, Scott, Hicks, Wilcox, Harris Munfakh, \& Shaffer, 2008; Scoliers et al., 2009). El bajo porcentaje de antecedente de intento suicida en la casuística estudiada podría deberse - al menos en parte - al bajo porcentaje de diagnóstico de trastorno mental $(28.07 \%)$ hallado en ella y a la casi ínfima presencia de comorbilidad psiquiátrica (sólo 5 casos de los $n=135$ ). La prevalencia de diagnóstico de trastorno mental hallada aquí fue mucho menor que antecedentes que reportaron que el $80 \%$ (Nock et al., 2010) o más del 90\% (Beautrais et al., 1996; Wunderlich et al., 1998) de quienes realizaron intentos suicidas padecía al menos un trastorno mental al momento de la tentativa, estando afectados por diagnósticos mentales comórbidos en el 56.6\% (Beautrais et al., 1996) al 79\% (Wunderlich et al., 1998) de los casos. Por otra parte, en el estudio realizado, la presencia de diagnóstico de trastorno mental caracterizó diferencialmente a pacientes masculinos antes que a femeninos. En su conjunto, los resultados hallados hasta aquí condicen con conclusiones que señalan que las mujeres tienen mayores tasas de intento suicida independientemente del número de diagnósticos psiquiátricos (Móscicki, 1994). No obstante, al incluir el análisis del marcador demográfico edad, la presencia de diagnóstico de trastorno mental caracterizó diferencialmente a casos femeninos y del grupo ambos sexos de 35-44 y 55-64 años y la ausencia de esos diagnósticos a los de 15-24 años. A este respecto cabe mencionar que en un trabajo anterior demostramos que intento de suicidio es un motivo de consulta psicológica en urgencias que caracteriza diferencialmente a los casos de 15-24 años en ambos sexos (López Steinmetz, 2017).

Tomados en conjunto, estos resultados sugieren que la asociación intento de suicidio-edad adolescente no podría ser atribuida primordialmente a enfermedad mental tal como propone alguna bibliografía (Ferreri, Agbokou, Nuss, \& Peretti, 2006), y distan de revisiones que señalan que la presencia de trastornos mentales afecta hasta al $80 \%$ de adolescentes que intentan suicidarse (Bridge et al., 2006). No obstante, para profundizar la discusión de estos tópicos son necesarias nuevas investigaciones locales, ya que la mayoría de las referencias se basan en poblaciones no-latinas a pesar de que hay estudios que advierten sobre diferencias en la conducta suicida entre latinos y no-latinos. Por caso, determinadas construcciones culturales en latinos parecen proporcionar protección contra la conducta suicida en presencia de enfermedad mental (Oquendo et al., 2005). 
Dos limitaciones deben ser tenidas en cuenta al interpretar los resultados hallados según Tipos y Categorías diagnósticas. Una, es la alta frecuencia de casos agrupados como Diagnóstico desconocido, y la otra, es el margen de error de los cálculos de $\chi^{2}$ debido a las bajas frecuencias de muchos diagnósticos. Aún a pesar de ello, lo hallado armoniza con antecedentes que destacaron entre los trastornos mentales más frecuentes en intentos suicidas a Trastornos del estado de ánimo, especialmente depresivos, y a Trastornos relacionados con sustancias (Beautrais, 2000; Bridge et al., 2006; Teti et al., 2014). Además, en la casuística estudiada se hallaron diferencias significativas en la distribución de las Categorías diagnósticas según sexo de los pacientes. Un interrogante que se sigue de lo hallado es saber si esas caracterizaciones son específicas de intento suicida o, por el contrario, si son consecuencia de diferencias de sexo en la prevalencia de trastornos mentales en las consultas psicológicas en urgencias, o bien, en la población general. Nuevamente, dar respuesta a esa importante cuestión requerirá del desarrollo de nuevas investigaciones locales. No obstante ello, lo hallado aún objeta el señalamiento de un predominio femenino entre pacientes que realizan intentos suicidas y que padecen Trastornos afectivos, incluyendo Depresión (Arsenault-Lapierre, Kim, \& Turecki, 2004), ya que al contrario de ello, los resultados hallados armonizan con revisiones que destacan que dichos trastornos mentales son los más frecuentes en intentos suicidas y suicidios de ambos sexos (Hawton, Casañas i Comabella, Haw, \& Saunders, 2013; Teti et al., 2014).

En cambio, las diferencias según sexo halladas en este estudio van en el sentido de otros estudios que destacaron que los intentos suicidas son relativamente frecuentes en pacientes con Trastornos de la conducta alimentaria (Franko \& Keel, 2006; Pompili, Girardi, Tatarelli, Ruberto, \& Tatarelli, 2006; Portzky, Van Heeringen, \& Vervaet, 2014), y que entre sujetos diagnosticados de Esquizofrenia, el sexo masculino - además de otros factores - confiere mayor riesgo de conducta suicida (Carlborg, Winnerbäck, Jönsson, Jokinen, \& Nordström, 2010). En contraste con lo hallado según sexo, no se encontraron diferencias significativas entre Categorías diagnósticas y edad de los pacientes. Al respecto, hay señalamientos de que los trastornos mentales más frecuentemente asociados a conductas suicidas en adolescentes y adultos jóvenes son Trastornos del estado de ánimo, Trastornos relacionados con sustancias y Trastornos de conducta (Beautrais, 2000; Fergusson, Woodward, \& Horwood, 2000). En la casuística analizada, si bien las dos primeras de esas Categorías diagnósticas acumularon frecuencias elevadas en la franja etaria 15-24 años, también lo hicieron en 25-34 y 35-44 años.

El $56.30 \%$ de los pacientes que tenían diagnóstico de trastorno mental recibía tratamiento a causa del diagnóstico al momento del intento suicida. Estudios que examinaron el grado de cumplimiento e incumplimiento terapéutico en pacientes psiquiátricos y los factores que lo afectan encontraron porcentajes similares. Informaron cumplimiento terapéutico en el $58.2 \%$ de los pacientes, y entre las razones más destacadas de incumplimiento terapéutico estuvo el desconocimiento de aspectos referidos al tratamiento (Bener et al., 2013). En efecto, es ampliamente reconocido que proporcionar información al paciente es un pilar base para conseguir el cumplimiento terapéutico (Basterra Gabarró, 1999). Con fundamento en ello y en los resultados hallados, se sugiere que el importante porcentaje de desinformación acerca del diagnóstico - reflejado por la Categoría establecida como Diagnóstico desconocido, que afectó al $28.15 \%$ de los pacientes con trastorno mental -, habría contribuido al alto porcentaje de casos que no recibía tratamiento al momento del intento suicida $(43.70 \%)$ y que se puede caracterizar por incumplimiento terapéutico. Además de la falta de información, las características de la enfermedad se cuentan entre otros tantos factores que influyen en el incumplimiento terapéutico (Basterra Gabarró, 1999), el que parece ser más frecuente en determinados diagnósticos psiquiátricos, principalmente en Trastornos depresivos (Bener et al., 2013; DiMatteo et al., 2000), bipolares (Gaudiano et al., 2008) y en Esquizofrenia (Bener et al., 2013).

En pacientes con Trastorno depresivo mayor, la probabilidad de realizar intento suicida se estima que es aproximadamente el doble entre los que tienen baja adherencia (Ruengorn et al., 2012). Llamativamente, en la presente investigación el porcentaje de casos que no recibía tratamiento al momento del intento suicida $(43.70 \%)$ fue casi equivalente al porcentaje que resulta de sumar los casos que desconocían el diagnóstico (28.15\%) y los casos con diagnóstico de Trastorno depresivo (16.30\%). No obstante, otros aspec- 
tos que también deben considerarse al interpretar los resultados son las diferencias observadas según marcadores demográficos de riesgo suicida. Similar a lo hallado aquí, por una parte, otros estudios reportaron que en comparación con los hombres, las mujeres son más propensas a realizar consultas médicas con especialistas de salud mental (Shapiro et al., 1984), aunque un estudio más reciente halló que si bien los hombres eran menos propensos que las mujeres a percibir la necesidad de tratamiento no obstante tenían la misma probabilidad de aceptar recibir tratamiento de salud mental (Narendorf \& Palmer, 2016), y por otra parte, se reportó que entre pacientes psiquiátricos, los de 21-30 años de edad se caracterizan por mayor cumplimiento terapéutico (Bener et al., 2013). Por lo demás, la complejidad de los múltiples factores implicados en el incumplimiento terapéutico requiere que aspectos tales como accesibilidad y disponibilidad de servicios de salud, entre otros, sean incluidos en análisis futuros.

\section{Referencias}

Álvarez Cáceres, R. (2007). Estadística aplicada a las ciencias de la salud. Madrid: Ediciones Díaz de Santos.

Arsenault-Lapierre, G., Kim, C. \& Turecki, G. (2004). Psychiatric diagnoses in 3275 suicides: a meta-analysis. BioMed Central Psychiatry, 4(37). Recuperado de http://www.biomedcentral.com/1471-244X/4/37. doi: $10.1186 / 1471-244 \mathrm{x}-4-37$

Asociación Americana de Psiquiatría (2002). Manual Diagnóstico y Estadístico de los Trastornos Mentales, texto revisado $(D S M-I V-T R)$. Buenos Aires: Masson.

Baldessarini, R. J., Pompili, M., \& Tondo, L. (2006). Suicide in bipolar disorder: Risks and management. CNS Spectrums, 11(6), 465-471. doi: 10.1017/ S1092852900014681

Basterra Gabarró, M. (1999). El cumplimiento terapéutico. Pharmaceutical Care España, 1, 97-106.

Beautrais, A. L. (2000). Risk factors for suicide and attempted suicide among young people. Australian and New Zealand Journal of Psychiatry, 34(3), 420-436. doi: 10.1007/0-306-47150-7 23

Beautrais, A. L. (2001). Suicides and serious suicide attempts: Two populations or one? Psychological Medicine, 31(5), 837-845. doi: 10.1017/s0033291701003889

Beautrais, A. L., Joyce, P. R., Mulder, R. T., Fergusson, D. M., Deavoll, B. J., \& Nightingale, S. K. (1996). Prevalence and comorbidity of mental disorders in persons making serious suicide attempts: A case-control study. The American Journal of Psychiatry, 153(8), 10091014. doi: 10.1176/ajp.153.8.1009

Bener, A., Dafeeah, E. E., \& Salem, M. O. (2013). A study of reasons of non-compliance of psychiatric treatment and patients' attitudes towards illness and treatment in Qatar. Issues in Mental Health Nursing, 34(4), 273280. doi: 10.3109/01612840.2012.745039
Bolton, J. M., Pagura, J., Enns, M. W., Grant, B. \& Sareen, J. (2010). A population-based longitudinal study of risk factors for suicide attempts in major depressive disorder. Journal of Psychiatric Research, 44(13), 817-826. doi: 10.1016/j.jpsychires.2010.01.003

Brent, D. A., Perper, J. A., Goldstein, C. E., Kolko, D. J., A1lan, M. J., Allman, C. J., \& Zelenak, J. P. (1988). Risk factors for adolescent suicide: A comparison of adolescent suicide victims with suicidal inpatients. Archives of General Psychiatry, 45(6), 581-588. doi: 10.1001/ archpsyc.1988.01800300079011

Bridge, J. A., Goldstein, T. R., \& Brent, D. A. (2006). Adolescent suicide and suicidal behavior. Journal of Child Psychology and Psychiatry, and Allied Disciplines, 47(3-4), 372-394. doi: 10.1111/j.14697610.2006.01615.x

Carlborg, A., Winnerbäck, K., Jönsson, E. G., Jokinen, J. \& Nordström, P. (2010). Suicide in schizophrenia. Expert Review of Neurotherapeutics, 10(7), 1153-1164. doi: 10.1586/ern. 10.82

Colborn, V. A., La Croix, J. M., Neely, L. L., Tucker, J., Perera, K., Daruwala, S. E.,... Ghahramanlou-Holloway, M. (2017). Motor impulsivity differentiates between psychiatric inpatients with multiple versus single lifetime suicide attempts. Psychiatry Research, 253, 1821. doi: 10.1016/j.psychres.2017.03.026

Diekstra, R. F. \& Gulbinat, W. (1993). The epidemiology of suicidal behavior: A review of three continents. World Health Statistics Quarterly, 46(1), 52-68.

DiMatteo, M. R., Lepper, H. S., \& Croghan, T. W. (2000). Depression is a risk factor for noncompliance with medical treatment. Archives of Internal Medicine, 160(14), 2101-2107. doi: 10.1001/archinte.160.14.2101

Fergusson, D. M., Woodward, L. J., \& Horwood, L. J. (2000). Risk factors and life processes associated with the onset of suicidal behavior during adolescence and early adulthood. Psychological Medicine, 30(1), 23-39. doi: 10.1017/s003329179900135x

Ferreri, F., Agbokou, C., Nuss, P., \& Peretti, C. S. (2006). Clinique des états dépressifs. Encyclopédie MédicoChirurgicale - Psychiatrie, E-37-110-A-10, 1-21.

Forman, E. M., Berk, M. S., Henriques, G. R., Brown, G. K., \& Beck, A. T. (2004). History of multiple suicide attempts as a behavioral marker of severe psychopathology. The American Journal of Psychiatry, 161(3), 437-443. doi: 10.1176/appi.ajp.161.3.437

Franko, D. L. \& Keel, P. K. (2006). Suicidality in eating disorders: Occurrence, correlates, and clinical implications. Clinical Psychology Review, 26(6), 769-782. doi: 10.1016/j.cpr.2006.04.001

Gaudiano, B. A., Weinstock, L. M., \& Miller, I. W. (2008). Improving treatment adherence in bipolar disorder: A review of current psychosocial treatment efficacy and recommendations for future treatment development. Behavior Modification, 32(3), 267-301. doi: 10.1177/0145445507309023

González Pinto, A., Mosquera, F., Alonso, M., López, P., Ramírez, F., Vieta, E., \& Baldessarini, R. J. (2006). Suicide risk in bipolar I disorder patients and adherence to long-term lithium treatment. Bipolar Disorders, $5(\mathrm{Pt}$ 2), 618-624. doi: 10.1111/j.1399-5618.2006.00368.x

Groholt, B. \& Ekeberg, O. (2009). Prognosis after adolescent suicide attempt: Mental health, psychiatric treatment, and suicide attempts in a nine-year follow-up study. Suicide \& Life-Threatening Behavior, 39(2), 125-136. doi: 10.1521/suli.2009.39.2.125 
Harris, E. C. \& Barraclough, B. (1997). Suicide as an outcome for mental disorders: A meta-analysis. The British Journal of Psychiatry, 170(3), 205-228. doi: 10.1192/bjp.170.3.205

Haukka, J., Suominen, K., Partonen, T., \& Lönngvist, J. (2008). Determinants and outcomes of serious attempted suicide: A nationwide study in Finland, 19962003. American Journal of Epidemiology, 167(10), 1155-1163. doi: 10.1093/aje/kwn017

Hawton, K., Casañas i Comabella, C., Haw, C. \& Saunders, K. (2013). Risk factors for suicide in individuals with depression: A systematic review. Journal of Affective Disorders, 147(1-3), 17-28. doi: 10.1016/j.jad.2013.01.004

Hawton, K., Sutton, L., Haw, C., Sinclair, J., \& Deeks, J. J. (2005). Schizophrenia and suicide: Systematic review of risk factors. The British Journal of Psychiatry, 187, 9-20. doi: 10.1192/bjp.187.1.9

Hawton, K. \& Van Heeringen, K. (2009). Suicide. The Lancet, 373(9672), 1372-1381. doi: 10.1016/s01406736(09)60372-x

Hishinuma, E. S., Smith, M. D., McCarthy, K., Lee, M., Goebert, D. A., Sugimoto-Matsuda, J. J.,... Andrade, J. K. L. (2017). Longitudinal prediction of suicide attempts for a diverse adolescent sample of native Hawaiians, Pacific peoples, and Asian Americans. Archives of Suicide Research, 10 Jan 2017, 1-24. doi: 10.1080/13811118.2016.1275992

Johnson Fridell, E., Ojehagen, A., \& Träskman-Bendz, L. (1996). A 5-year follow-up study of suicide attempts. Acta Psychiatrica Scandinavica, 93(3), 151-157. doi : 10.1111/j.1600-0447.1996.tb10622.x

Joiner, T. E. Jr. \& Rudd, M. D. (2000). Intensity and duration of suicidal crises vary as a function of previous suicide attempts and negative life events. Journal of Consulting and Clinical Psychology, 68(5), 909-916. doi: 10.1037//0022-006x.68.5.909

Joiner, T. E. Jr., Rudd, M. D., Rouleau, M. R., \& Wagner, K. D. (2000). Parameters of suicidal crises vary as a function of previous suicide attempts in youth inpatients. Journal of the American Academy of Child and Adolescent Psychiatry, 39(7), 876-880. doi: 10.1097/00004583-200007000-00016

Kulkarni, R. R., Rao, K. N., \& Begum, S. (2013). Comorbidity of psychiatric and personality disorders in first suicide attempters: a case-control study. Asian Journal of Psychiatry, 6(5), 410-416. doi: 10.1016/j. ajp.2013.05.004

López Steinmetz, L. C. (2015). Marcadores temporales de riesgo supuesto en casos asistidos psicológicamente en un Servicio de Guardia: Descripción de sus distribuciones y confrontación de la hipótesis de asociación entre temporalidad y conductas suicidas. Investigaciones en Psicología, 20(2), 79-93.

López Steinmetz, L. C. (2017). Caracterización de riesgo diferencial-demográfico en urgencias psicológicas: intento de suicidio y otros motivos de consulta. Revista Argentina de Ciencias del Comportamiento, 9(1), 44-53.

López Steinmetz, L. C. (n.d.). Coyunturas desencadenantes de urgencias suicidas: un enfoque psicoanalítico y epidemiológico (Tesis de Doctorado). Buenos Aires: Universidad de Buenos Aires, Facultad de Psicología.

Miranda, R., Scott, M., Hicks, R., Wilcox, H. C., Harris Munfakh, J. L., \& Shaffer, D. (2008). Suicide attempts characteristics, diagnoses, and future attempts: Comparing multiple attempters to single attempters and ideators. Journal of the American Academy of Child and Adolescent Psychiatry, 47(1), 32-40. doi: 10.1097/ chi.0b013e31815a56cb
Móscicki, E. K. (1994). Gender differences in completed and attempted suicides. Annals of Epidemiology, 4(2), 152-158. doi: 10.1016/1047-2797(94)90062-0

Narendorf, S. C. \& Palmer, A. (2016). Perception of need and receipt of mental health treatment: A three-group comparison of young adults with psychological distress. Psychiatric Services, 67(8), 924-927. doi: 10.1176/ appi.ps.201500230

Nock, M. K., Borges, G., Bromet, E. J., Cha, C. B., Kessler, R. C., \& Lee, S. (2008). Suicide and suicidal behavior. Epidemiologic Reviews, 30(1), 133-154. doi: 10.1093/ epirev/mxn002

Nock, M. K., Hwang, I., Sampson, N. A., \& Kessler, R. C. (2010). Mental disorders, comorbidity and suicidal behavior: Results from the National Comorbidity Survey Replication. Molecular Psychiatry, 15(8), 868-876. doi: $10.1038 / \mathrm{mp} .2009 .29$

Nock, M. K., Hwang, I., Sampson, N. A., Kessler, R. C., Angermeyer, M., Beautrais, A.,... Williams, D. R. (2009). Cross-national analysis of the associations among mental disorders and suicidal behavior: Findings from the WHO World Mental Health Surveys. PLoS Medicine, 6(8), e1000123. doi: 10.1371/journal. pmed. 1000123

O’Carroll, P. W., Berman, A. L., Maris, R. W., Moscicki, E. K., Tanney, B. L., \& Silverman, M. M. (1996). Beyond the Tower of Babel: A nomenclature for suicidology. Suicide \& Life-Threatening Behavior, 26(3), 237-252.

Oquendo, M. A., Dragatsi, D., Harkavy-Friedman, J., Dervic, K., Currier, D., Burke, A. K.,... Mann, J. J. (2005). Protective factors against suicidal behavior in Latinos. The Journal of Nervous and Mental Disease, 193(7), 438-443. doi: 10.1097/01.nmd.0000168262.06163.31

Organización Mundial de la Salud (2014). Prevención del suicidio: Un imperativo global. Ginebra: Organización Mundial de la Salud.

Polewka, A., Groszek, B., Targosz, D., Szkolnicka, B., Chrostek Maj, J., Kroch, S., \& Pach, J. (2004). The frequency of suicide attempts depending on gender and the age structure. Przeglad Lekarski, 61(4), 265-268.

Pompili, M., Girardi, P., Tatarelli, G., Ruberto, A., \& Tatarelli, R. (2006). Suicide and attempted suicide in eating disorders, obesity and weight-image concern. Eating Behaviors, 7(4), 384-394. doi: 10.1016/j.eatbeh.2005.12.004

Porta, M. S. (Ed.) (2014). A dictionary of epidemiology (6th ed.). New York, United States of America: Oxford University Press.

Portzky, G., Van Heeringen, K., \& Vervaet, M. (2014). Attempted suicide in patients with eating disorders. Crisis, 35(6), 378-387. doi: 10.1027/0227-5910/a000275

Ribeiro, J. D., Franklin, J. C., Fox, K. R., \& Bentley, K. H. (2016). Self-injurious thoughts and behaviors as risk factors for future suicide ideation, attempts, and death: A meta-analysis of longitudinal studies. Psychological Medicine, 46(2), 225-236. doi: 10.1017/ s0033291715001804

Ruengorn, C., Sanichwankul, K., Niwatananun, W., Mahatnirunkul, S., Pumpaisalchai, W., \& Patumanond, J. (2012). A risk-scoring scheme for suicide attempts among patients with bipolar disorder in a Thai patient cohort. Psychology Research and Behavior Management, 5, 37-45. doi: 10.2147/prbm.s30878

Schmidtke, A., Bille-Brahe, U., Deleo, D., Kerkhof, A., Bjerke, T., Crepet, P.,... Sampaio-Faria, J. G. (1996). Attempted suicide in Europe: Rates, trends and sociodemographic characteristics of suicide attempters during the period 1989-1992. Results of the WHO/EURO 
Multicentre Study on Parasuicide. Acta Psychiatrica Scandinavica, 93(5), 327-338. doi: 10.1111/j.16000447.1996.tb10656.x

Scoliers, G., Portzky, G., Van Heeringen, K., \& Audenaert, K. (2009). Sociodemographic and psychopathological risk factors for repetition of attempted suicide: A 5-year follow-up study. Archives of Suicide Research, 13(3), 201-213. doi: 10.1080/13811110902835130

Shapiro, S., Skinner, E. A., Kessler, L. G., Von Korff, M., German, P. S., Tischler, G. L.,... Regier, D. A. (1984). Utilization of health and mental health services: Three Epidemiologic Catchment Area sites. Archives of General Psychiatry, 41(10), 971-978. doi: 10.1001/archpsyc. 1984.01790210053007

Suominen, K., Isometsä, E., Suokas, J., Haukka, J., Achte, K., \& Lönngvist, J. (2004). Completed suicide after a suicide attempt: A 37-year follow-up study. The American Journal of Psychiatry, 161(3), 562-563. doi: 10.1176/appi.ajp.161.3.562
Teti, G. L., Rebok, F., Rojas, S. M., Grendas, L., \& Daray, F. M. (2014). Systematic review of risk factors for suicide and suicide attempt among psychiatric patients in Latin America and Caribbean. Revista Panamericana de Salud Pública, 36(2), 124-133.

Wunderlich, U., Bronisch, T., \& Wittchen, H. U. (1998). Comorbidity patterns in adolescents and young adults with suicide attempts. European Archives of Psychiatry and Clinical Neuroscience, 248(2), 87-95. doi: 10.1007/s004060050023

Yoshimasu, K., Kiyohara, C., Miyashita, K., \& The Stress Research Group of the Japanese Society for Hygiene (2008). Suicidal risk factors and completed suicide: Meta-analyses based on psychological autopsy studies. Environmental Health and Preventive Medicine, 13(5), 243-256. doi: 10.1007/s12199-008-0037-x

Para citar este artículo:

López Steinmetz, L. C. (2017). Factores psicopatológicos de riesgo en intentos de suicidio. Ciencias Psicológicas, 11(1), 89 - 100. doi: $10.22235 /$ cp.v11i2.1350 